1

2

3

\title{
Effects of positive mood on probabilistic learning: behavioral and
} electrophysiological correlates

(1) Department of Experimental Clinical \& Health Psychology, Ghent University, Ghent, Belgium

(2) Department of Psychology and Neuroscience, University of Colorado Boulder, USA

Correspondence :

Gilles Pourtois

Jasmina Bakic $^{1}$, Marieke Jepma ${ }^{2}$, Rudi De Raedt ${ }^{1}, \&$ Gilles Pourtois ${ }^{1}$

Department of Experimental-Clinical and Health Psychology

Ghent University

Henri Dunantlaan 2

9000 Gent, Belgium

Phone: +3292649144

Email: gilles.pourtois@ugent.be 


\begin{abstract}
Whether positive mood can change reinforcement learning or not remains an open question. In this study, we used a probabilistic learning task and explored whether positive mood could alter the way positive versus negative feedback was used to guide learning. This process was characterized both at the behavioral and electro-encephalographic levels. Thirty two participants were randomly allocated either to a positive or a neutral (control) mood condition. Behavioral results showed that while learning performance was balanced between the two groups, participants in the positive mood group had a higher learning rate than participants in the neutral mood group. At the electrophysiological level, we found that positive mood increased the errorrelated negativity when the stimulus-response associations were deterministic, selectively (as opposed to random or probabilistic). However, it did not influence the feedback-related negativity. These new findings are discussed in terms of an enhanced internal reward prediction error signal after the induction of positive mood when the probability of getting a reward is high.
\end{abstract}


The influential "broaden and build" theory put forward by Fredrickson (2004) stipulates that positive emotion is associated with a variety of beneficial changes regarding emotional and cognitive functioning (Fredrickson, 2004). Among them, positive mood might shield the organism from experiencing high levels of negative affect (or anxiety) during the encounter of punishment or worse than expected outcomes. Conversely, it might foster reward processing in the face of positive or favorable outcomes. Previous research already showed that depression (characterized by a lack of positive affect or anhedonia) influences these two processes concurrently (Eshel \& Roiser, 2010). Moreover, earlier studies and models already emphasized that positive affect fuels problem solving, creativity as well as cognitive control, suggesting that its effect on high-level cognition might be domain general, as opposed to valence specific (for a review, see Ashby, Isen, \& Turken, 1999; Garland et al., 2010). In other words, positive mood might influence specific cognitive processes, with or without producing a selective change in the way either reward or punishment is eventually processed. Furthermore, positive mood might play an important role in well-being and contribute to tip the balance of emotional processing by downplaying negative affect, while concurrently up-regulating the weight of positive affect. However, experimental evidence corroborating this assumption is scant. An elegant means to assess how positive mood might dynamically influence emotional processing (when positive and negative reinforcers are in direct competition in the environment and are therefore deemed equally important events to guide behavior) is provided by probabilistic learning tasks.

During probabilistic learning, the agent usually takes actions, and through trial and error, he/she tries to decipher associations linking stimuli to rewards (Frank, Woroch, \& Curran, 2005). Feedback (being either a reward or a punishment) is provided following each action to signal 
whether this action was correct or not. This typically results in an increase in rewarded actions and a decrease in punished actions over time, which is known as the law of effect in reinforcement learning (RL) model (Walsh \& Anderson, 2011). Updating of the response pattern based on the reward and punishment can be traced by the learning rate parameter $(\alpha)$, which reflects current levels of uncertainty about one's choice strategies, and reliance on the history of previous responses (Behrens, Woolrich, Walton, \& Rushworth, 2007). Whether or not positive emotion can reliably influence these processes remains an open issue. Earlier research already showed that positive mood could be beneficial (compared to neutral or negative mood) to cognitive flexibility and in turn learning performance (when it is primarily rule-based or rule described; see Nadler et al., 2010). However, it is still unclear whether these changes in learning following the induction of positive mood may be explained by a differential sensitivity to cues signaling (violation of) reward.

To address this question, in this study, we used a previously validated mood induction procedure (Vanlessen, Rossi, De Raedt, \& Pourtois, 2013) to induce, using a between-subjects design, either a positive or neutral (control) mood, which was then maintained successfully throughout the entire experimental session by means of several rehearsals. Participants performed a variant of the probabilistic learning task previously devised by Eppinger, Kray, Mock, and Mecklinger (2008), while electroencephalogram (EEG) was recorded continuously in order to explore changes in the error and feedback related negativity (ERN and FRN) components depending on learning and mood. We chose this specific task setting (see also Frank et al., 2005) because it allows us to explore and characterize mood-related (neurophysiological) changes occurring either at the response or feedback level across different conditions varying in reward probability and shown in random order (Eppinger et al., 2008). These two ERP components are 
traditionally related to error processing based on internal (motor) and external cues, respectively. The ERN/Ne is a negative deflection over fronto-central electrodes peaking $\sim 0-100 \mathrm{~ms}$ after the onset of an incorrect response, hence is based on an internal (motor) representation (Falkenstein et al., 1991; Falkenstein et al., 2000; Carbonnell \& Falkenstein, 2006; Gehring, Goss, Coles, Meyer, \& Donchin, 1993). By contrast, the FRN is thought to be its feedback-related counterpart, appearing roughly 250-400 $\mathrm{ms}$ after the presentation of negative feedback (Gehring \& Willoughby, 2002; Holroyd \& Coles, 2002; Miltner, Braun, \& Coles, 1997).

In our study, we examined mood effects on learning in this task using (i) standard behavioral data (i.e., speed and accuracy of the responses, as in Eppinger et al., 2008), and (ii) estimated learning-rate parameters extracted from a reinforcement-learning (RL) model (Sutton \& Barto, 1998) with separate learning rates for positive and negative reinforcement (e.g., Frank, Moustafa, Haughey, Curran, \& Hutchison, 2007). Two separate sets of predictions were made. At the behavioral level, we expected that positive mood might alter learning during this task. More specifically, because positive mood might fuel reward processing (Garland et al., 2010) and it shares common neurobiological ground with it (Ashby et al., 1999), we surmised that participants in the positive mood group would be more sensitive to positive feedback than participants in the neutral mood group. Accordingly, learning rate following positive feedback could be higher in the positive, compared to the neutral mood group. At the electrophysiological level, because both the ERN and FRN components have been linked to reward prediction error (Holyroyd \& Coles, 2002, Nieuwenhuis, Slagter, von Geusau, Heslenfeld, \& Holroyd, 2005), we predicted that positive mood might influence the size of these ERP components (see also Lange, Leue, \& Beauducel, 2012), in opposite situations however. More specifically, we predicted that positive mood could increase the magnitude of the ERN component (coding reward prediction error at the 
response level) when learning was deemed feasible during the probabilistic learning task (Eppinger et al., 2008), that is when (motor) cues signaling violation of reward were actually informative to guide learning (i.e., they conveyed relevant information about the current goal conduciveness of the action performed). By contrast, when learning was made impossible (because feedback cues signaling reward or punishment appeared to be random and/or unrelated to the actual action performed), we surmised that positive mood could increase the size of the FRN component (coding reward prediction error at the feedback level). Translated to the task previously devised by Eppinger et al. (2008) and used here, we therefore hypothesized that positive mood (compared to neutral mood) could increase the ERN when the clarity and certainty regarding the stimulus-response associations were high ("deterministic" or rule-based learning; see also Nalder et al., 2010). Conversely, we expected positive mood to augment the FRN component when the clarity and certainty regarding the stimulus-response associations was low ("probabilistic" learning or random condition; see also Lange, Leue, \& Beauducel, 2012). Hence, following standard practice (see Frank et al., 2005), we conceived the ERN and FRN components as valid electrophysiological markers of violation of reward. If confirmed, these results might lend support to the assumption that positive mood could influence RL by changing the sensitivity to both motor (ERN) and feedback (FRN) cues signaling violation of reward (Garland et al., 2010). 


\section{METHODS}

\section{Participants}

Thirty eight participants (undergraduate psychology students) took part in the study in exchange of 30 Euro compensation. They were randomly assigned to one of two groups: positive vs. neutral mood. They were all right-handed, with no past or current neurological or psychiatric problems, they had normal or corrected-to normal vision, and all gave written informed consent prior to the start of the experiment. The data of six participants were removed according to the following exclusion criteria. First, participants in the positive group had to show a marked increase in positive mood following the mood induction compared to the baseline (i.e., the average increase had to be above the baseline value, and there should be no decrease), while no change in positive mood was expected to take place in the neutral group. In this group, two participants were excluded since their average happiness level was higher than the mean of the positive group. Second, participants showing no learning during the main task (i.e., their learning curves did not differ from chance level) were excluded ( $n=3$; two in the positive and one in the neutral mood group). The behavioral results obtained for the accuracy, RT and learning rate data still remained significant when including them in the statistical analyses. However, because they did not show learning, their data were deemed noisy and were therefore removed from the subsequent statistical analyses. Finally, the data of one participant was excluded because of technical problems during the recording of the EEG. The final sample consisted of 32 participants ( mean age $=22.3$ years, S.D. $=2.4,25$ females), 16 in each mood group. The study was approved by the local ethics committee. 


\section{Mood induction}

We used a previously validated mood induction procedure (MIP; see Vanlessen, et al., 2013, 2014). Mood was induced by means of a guided imagery procedure, where participants were instructed to vividly imagine reliving either a happy or neutral (depending on the group they were assigned to) autobiographical memory (Holmes, Coughtrey, \& Connor, 2008; Holmes, Mathews, Dalgleish, \& Mackintosh, 2006). First, the participants were trained in taking a field perspective (i.e., imagining from one's own perspective) during mental imagery. Then they had to choose an appropriate happy/neutral event, an episodic memory that happened at least a week before, and report about it. For the recall that would ensue, they were instructed to keep their eyes closed and visualize all the specificities of the memory, and to use the field perspective (Watkins \& Moberly 2009, based on Holmes et al., 2008). The actual recall session was divided into two parts of $30 \mathrm{~s}$, and in between participants were asked questions about different aspects of the happy/neutral memory they were imagining. Participants were blind to the real purpose of the procedure, believing that it was about remembering an event from the past as vividly as possible (and not about re-experiencing the emotion of the event). After each mood induction, participants marked on 10-cm horizontal visual analogue scales (VAS) their current level of happiness, pleasantness, and sadness, with "neutral" on one end/anchor to "as happy/pleasant/sad as I can imagine" on the other.

\section{Probabilistic learning task}

We used a probabilistic learning task previously validated by Eppinger et al. (2008). In this task, participants were asked to decipher and learn, by trial and error, several hidden stimulus-response (S-R) mappings. For each trial, participants were asked to decide, with a time 
limit, whether the stimulus shown on the screen was associated with response 1 or 2 . Feedback on the choice made by the participant was given following every response made.

Participants were presented with 6 visual stimuli (A-B-C-D-E-F), belonging to three conditions (unknown to the participants) that differed regarding the actual probability of the S-R mapping $(100 \%, 80 \%$ or $50 \%)$. In the condition $100 \%$, each stimulus of the pair was always associated with one of the two response keys, corresponding to a "deterministic" S-R mapping (i.e., response 1 was always correct for stimulus A, and response 2 for the stimulus B). In the condition $80 \%$, the S-R mapping was "probabilistic", given that stimulus C was associated $80 \%$ of the time with response 1 (and thus $20 \%$ of the time with the concurrent response 2), while stimulus D had a symmetric probability for the S-R mapping. Finally, in the condition $50 \%$ ("random" S-R mapping), each stimulus of the pair was associated equally often to each of the two response keys (i.e., stimuli $\mathrm{E}$ and $\mathrm{F}$ were associated 50\% of the time with response 1 and $50 \%$ of the time with response 2 ).

Colorful line drawings (Rossion \& Pourtois, 2004) were used as visual stimuli, presented against a white homogenous background on a 17-inch computer screen. These stimuli were visual objects belonging to different semantic categories (artifacts, buildings, musical instruments, clothes, vehicles, furniture). Their mean size was $7 \mathrm{~cm}$ width $\mathrm{x} 5 \mathrm{~cm}$ height, corresponding to $5 \mathrm{x}$ 3,6 degrees of visual angle at $80 \mathrm{~cm}$ viewing distance.

The trial structure was as follows: it began with a fixation cross of $250 \mathrm{~ms}$ duration. Then, the stimulus was presented for $500 \mathrm{~ms}$, followed by a blank screen of $300 \mathrm{~ms}$. Response deadline was set to $800 \mathrm{~ms}$ following the onset of the visual stimulus on the screen. Then, performance feedback was presented for $500 \mathrm{~ms}$. The feedback was provided in the form of a written word (in 


\begin{abstract}
Dutch) shown in black against a white homogenous background. This word was "goed" (correct), "fout" (incorrect), or "te traag" (too late). The inter-trial interval was constant (500 ms) and it corresponded to a blank screen, after which a new trial would ensue. Manual responses (i.e., key presses) were recorded using the Cedrus response box.
\end{abstract}

Each participant completed three blocks of 240 trials. Each block had six different stimuli, each repeated forty times. Accordingly, participants had to learn new S-R mappings in each block. Trial order within a block as well as the order of the three blocks were alternated across participants.

\title{
Procedure
}

First, participants were prepared for EEG recording. In order to get acquainted with the task, they completed a short practice session of 20 trials. Next, either a positive or neutral mood was induced by means of the MIP before the beginning of the first block. The same MIP was briefly rehearsed (5 minutes) halfway (after 120 trials) during the first block. The same procedure was repeated for the following two experimental blocks (i.e., the MIP was used each time at the beginning of the block and then rehearsed after the first half of trials was completed) in order to sustain the targeted mood throughout the whole experimental session. Hence, in total, participants encountered six times the MIP.

In order to strengthen the effect of positive mood, an evaluative feedback was added (rewarding in the positive mood group, and neutral in the neutral group) at the end of each block. This (bogus) feedback consisted of a small text shown on the screen, informing participants that they had to wait briefly until the computer had calculated online their learning performance up to that trial number. After a few seconds, an Excel-like scatter plot appeared on the screen, showing 
them their performance level allegedly relative to a group of peers. Their score was indicated by means of a color dot. This dot was positioned systematically either higher up in the distribution of scores for participants in the positive mood group, or somewhere in the middle of the distribution for those belonging to the neutral mood group. Next to this scatter plot, a specific written message was included. It informed them to try to keep the same level of performance. Manipulation checks of mood based on visual analog scales (see results here below) confirmed that this procedure (combined with the MIP) actually produced the desired effects: an increase of positive affect in the positive mood group, with no change in affect (neither positive, nor negative) in the neutral mood group. However, we have good reasons to believe that the change in positive mood (in the positive mood group) was mainly due to the MIP and the use of guided imagery (see also Vanlessen et al., 2013, 2014), and not so much to this (infrequent) feedback manipulation that occurred only three times during the course of the experiment. After each block, participants were asked to indicate, for each of the 6 stimuli, the clarity and certainty of each of the six stimulus-response (S-R) associations, by means of a horizontal 10-cm VAS. Furthermore, they were asked to rate the amount of positive vs. negative feedback they thought they received during this last block (using a $10 \mathrm{~cm}$ VAS going from "exclusively negative" to "exclusively positive"), as well as how much they liked or disliked these positive vs. negative feedback when receiving them (using a Likert scale spanning from 0 to 100).

Finally, participants were asked to fill out several trait-related questionnaires: the Beck Depression Inventory (Beck, Steer, Ball, \& Ranieri, 1996), the Resilience scale translated in Dutch (Portzky, De Bacquer, Audenaert, \& Wagnild, 2010), and the Frederickson's list of emotions (Fredrickson, 2001). The whole experiment lasted about 2 hours. 


\section{EEG recording}

EEG was recorded continuously using 64-channels by means of a Biosemi Active Two system (www. Biosemi.com). The EEG was sampled at $512 \mathrm{~Hz}$, with CMS-DRL serving as the reference-ground. The EEG signal was filtered off line, using a 0.016 to $70 \mathrm{~Hz}$ filter (12db/oct), with a $50 \mathrm{~Hz}$ notch and re-referenced using the linked (average) mastoids. Individual epochs were segmented using a -/+ $500 \mathrm{~ms}$ interval around the response (see Aarts \& Pourtois, 2010; Aarts et al., 2013; Pourtois, 2011), and -200/+1000ms around the onset of the visual feedback (see Aarts \& Pourtois, 2012). Eye blinks were removed automatically via vertical ocular correction (Gratton, Coles, \& Donchin, 1983), using two electrodes, placed above and below the right eye. Individual epochs were baseline corrected using the entire pre-stimulus time interval for the FRN (i.e., $200 \mathrm{~ms}$ ) and the first $200 \mathrm{~ms}$ of the pre-response time-interval for the ERN (i.e., from -500 to $-300 \mathrm{~ms}$ prior to response onset).

Artifact rejection was based on $\mathrm{a} \pm 100 \mu \mathrm{V}$ amplitude cutoff. For response locked segments, $87 \%$ of the individual segments were kept and eventually included in the averages. No significant group difference [positive: $\mathrm{M}=87.68$, $\mathrm{SEM}=4.8$; neutral $\mathrm{M}=86.42, \mathrm{SEM}=4.82$, $t(1,30)=0.73, p>.05]$ was found for this metric. For feedback-locked segments, 90\% of the individual epochs were kept. No group difference was found for this metric either [positive: $\mathrm{M=}$ 97.57, $\mathrm{SEM}=2.93$; neutral $: \mathrm{M}=83.42, \mathrm{SEM}=14.44, \mathrm{~F}(1,30)=1.74, \mathrm{p}>.05]$. Finally, individuals epochs were averaged separately for the different conditions and subjects, and an additional low pass filter set to $30 \mathrm{~Hz}$ was applied on the individual averages before grand-averaging. 
Mood manipulation. The efficiency of the change in positive (relative to neutral) mood following the MIP was assessed by means of mixed model ANOVAs with Group as between subject factor and Time $(n=7)$ as the within subject factor.

Accuracy analyses. Accuracy data were expressed in proportions of correct responses from the total number of trials, separately for each Condition $(n=3)$. Moreover, for each Condition separately (2 stimuli x 40 repetitions), changes of learning performance as a function of time were captured by grouping the data into four bins of equal size (i.e., 20 trials/condition) (see Eppinger et al., 2008 for a similar approach). These data were then submitted to a mixed model ANOVA with Group as between subject factor, and Condition and Bin as within subject factors.

Reinforcement-learning model. We fitted a basic Q-learning algorithm to each participant's choice data, using separate learning-rate parameters $\left(\propto_{+}\right.$and $\left.\propto_{-}\right)$for positive ("correct"; modeled as 1) and negative ("incorrect"; modeled as 0) feedback, respectively. These parameter estimates complement the accuracy data by informing about the participants' tendency to learn from positive vs. negative feedback.

On each trial, the model estimates the value $Q$ (i.e., probability of receiving "correct" feedback) associated with each of the 2 possible responses, separately for each stimulus. At the beginning of each block, the values of both responses for all stimuli were initialized to 0.5 . Then, each time that a participant selected response $r$ for stimulus $s$ on trial $t$, and received positive feedback (1), the estimated value of the chosen response for that stimulus was updated according to: 
$Q_{s, r, t+1}=Q_{s, r, t}+\propto_{+}\left[1-Q_{s, r, t}\right]$

Similarly, when the response resulted in negative feedback (0), the estimated value of the chosen response for that stimulus was updated according to:

$Q_{s, r, t+1}=Q_{s, r, t}+\propto_{-}\left[0-Q_{s, r, t}\right]$

The value of the other (unchosen) response associated with that stimulus was also updated (cf. Matsumoto, Matsumoto, Abe, \& Tanaka, 2007), such that the expected values of response 1 and response 2 associated with a given stimulus sum to 1 . Thus, if the value of the chosen response increases, the value of the unchosen response decreases with the same amount, and vice versa.

Choice behavior was modeled using the 'softmax' rule, with inverse-gain parameter $\beta$. With lower values of $\beta$ response selection is determined more by the relative estimated values of the two responses, whereas with higher values of $\beta$ response selection is more evenly distributed across the 2 responses (i.e., more random). The probability of choosing response 1 over response 2 for stimulus $s$ on trial $t,\left(P_{s, 1, t}\right)$, is computed as:

$$
P_{s, 1, t}=\frac{e^{\frac{Q_{s, 1, t}}{\beta}}}{e^{\frac{Q_{s, 1, t}}{\beta}}+e^{\frac{Q_{s, 2, t}}{\beta}}}
$$

We fitted the model to each participant's trial-by-trial choice sequences by maximizing the log-likelihood of the observed choices. We estimated the $\propto_{+}, \propto_{-}$and $\beta$ parameters for the 3 probability conditions together (deterministic, probabilistic and random, see above; see Walsh \& Anderson, 2014), resulting in 3 free parameters. We also fitted three other versions of the model and compared their goodness of fit using the Bayesian Information Criterion (BIC). First, we 
modeled the data using a shared learning rate for positive and negative feedback. Moreover, for each of these two models (using either separate or shared learning rates for positive and negative feedback), we modeled the updating of the two response options as either dependent or independent (see also results section below).

To optimize the parameter fits we used Matlab's fmincon function (Coleman et al., 1996), a constrained nonlinear optimization algorithm, with twenty randomized starting parameter estimates. The trials in which no response was made within the 800-ms time limit were omitted.

We submitted the best fitting $\propto_{+}$and $\propto_{-}$parameters to a mixed model ANOVA with group (neutral vs. positive mood) as between-subject factor and feedback valence (correct/ $\propto_{+}$vs. incorrect $\left./ \propto_{-}\right)$as within-subject factors. We also performed a group comparison on the best fitting $\beta$ parameter, using an independent samples t-test.

EEG analyses. We analyzed two well-documented error-related ERP components (Eppinger et al., 2008; Frank, et al., 2005; Mies et al., 2011). For the ERN, the mean amplitude was calculated in an interval spanning $100 \mathrm{~ms}$ after response onset at electrode FCz. For the FRN, we used a similar $100 \mathrm{~ms}$ time interval (centered around the peak; $50 \mathrm{~ms}$ prior and $50 \mathrm{~ms}$ after it) and calculated the mean amplitude of this component at the same fronto-central electrode (see Eppinger et al., 2008). The FRN peak was defined as the most negative deflection arising at electrode $\mathrm{FCz}$ in the 230-350 ms time window following feedback onset. Importantly, for the probabilistic condition, we only included trials corresponding to the dominant S-R mapping (80\%). Hence, epochs corresponding to the non-dominant S-R mapping (20\%) were removed from the averages giving rise to the FRN component in this condition (probabilistic condition). For each of these two deflections separately, a mixed-model ANOVA was performed on the 
average mean amplitudes with group as between subject and condition and response accuracy as within subject factors. In a second step, we computed difference waveforms by subtracting the ERP activity of incorrect from correct trials, separately for the ERN and FRN components, following standard practice (Eppinger et al., 2008).

\section{RESULTS}

\section{Behavioral Results}

Mood. The analysis of the MIP ratings (see Table 1) showed a significant Time*Group interaction for pleasantness $\left(F(6,180)=2.9, \mathrm{p}<.01, \eta^{2}=.1\right)$, happiness $\left(F(6,180)=6.6, p<.01, \eta^{2}=\right.$ $.2)$ and arousal $\left(F(6,180)=3.6, p<.01, \eta^{2}=.1\right)$. With the exception of the baseline measurement where no group difference arose, the positive group had a significantly higher level of pleasantness, happiness and arousal compared to the neutral mood group for almost all subsequent measurement points following the MIP. The two groups did not differ with regards to subjective levels of sadness ( $p>8)$

Accuracy. The number of too late responses was modest (mean proportion for the positive mood group: 2.68 , SD: 0.27 ; and for the neutral group: $2.85, \mathrm{SD}=0.27$ ) and did not differ between the two Groups, nor did it vary depending on condition or Bin (Bin 1: $\mathrm{M}=2.7, \mathrm{SD}=0.3$; Bin 2 : $\mathrm{M}=2.6, \mathrm{SD}=0.3$; Bin $3: \mathrm{M}=2.6, \mathrm{SD}=0.2$; Bin $4: \mathrm{M}=2.7, \mathrm{SD}=0.3$; all $\mathrm{p}$ 's $>0.9$ ). The analysis carried out on the proportions of correct responses (Figure 1) revealed significant main effects of Condition $\left(\mathrm{F}(2,60)=230.6, \mathrm{p}<.01, \eta^{2}=.9\right)$ and $\mathrm{Bin}\left(\mathrm{F}(3,90)=26.7, \mathrm{p}<.01, \eta^{2}=.5\right)$, as well as a significant Condition* Bin interaction $\left(F(6,180)=15.3, p<.01, \eta^{2}=.8\right)$ showing that learning curves differed across the three conditions. Learning was the quickest and accuracy the highest in 
the deterministic Condition, intermediate in the probabilistic Condition and merely absent in the random Condition. However, the factor Group did not interact with the main experimental factors (all p's >.1). The main effect of Group was not significant either $(\mathrm{p}=.3)$. Hence these standard accuracy data suggest a balanced learning across the two groups (Eppinger et al., 2008). The analysis performed on the RT data for correct responses revealed a significant main effect of Condition $\left(\mathrm{F}(2,60)=6.8, \mathrm{p}<.01, \eta^{2}=.2\right)$, while all the other effects were non-significant (all p's>.05). Follow up t-test showed faster RTs for the deterministic (Mean RT: 390.5, SD: 26.9; t $(31)=-2.8, \mathrm{p}<.01$,) and probabilistic Condition (Mean RT: 388.1, SD: 24.5, $\mathrm{t}(31)=-3.5, \mathrm{p}<.01$ ), compared to the random Condition (Mean RT: 399.5, SD: 30.0). RT between the deterministic and probabilistic Condition did not differ significantly $(\mathrm{t}(31)=.7, \mathrm{p}>.5)$.

We also extracted and analyzed the proportion of switches after negative feedback, as this metric has previously been related to exploration indirectly (e.g., Hills et al., 2010). This analysis confirmed that this rate varied significantly depending on reward probability/condition $(\mathrm{F}(2,60)=$ 23.1, $\left.\mathrm{p}<.01, \eta^{2}=.4\right)$. Switch rate after negative feedback was higher for the random $(\mathrm{M}=6.5$, $\mathrm{SD}=1.6)$ than either the deterministic $(\mathrm{M}=4.7, \mathrm{SD}=1.6 ; \mathrm{t}(31)=5.8, \mathrm{p}<.001)$ or the probabilistic condition $(\mathrm{M}=4.7, \mathrm{SD}=1.7 ; \mathrm{t}(31)=6.12, \mathrm{p}<.001)$. The difference between the deterministic and the probabilistic condition was not significant $(\mathrm{t}(31)=.07, \mathrm{p}=.94)$. This effect did not interact with group $/ \operatorname{mood}(\mathrm{F}(2,60)=1.4, \mathrm{p}=.25)$.

Learning rate. The ANOVA performed on the estimated learning-rate $(\alpha)$ parameters revealed significant main effects of Feedback Valence $\left(F(1,30)=116.65, p<.01, \eta^{2}=.7\right)$, and group $\left(\mathrm{F}(1,30)=6.2, \mathrm{p}<.05, \eta^{2}=.2\right)$, indicating that the learning rate was higher for positive than for negative feedback, and that the positive mood group had overall higher learning rates than the neutral mood group. Follow-up contrasts (using independent samples T-tests) showed a higher 

$\mathrm{SD}=0.10)$ following positive feedback $(\mathrm{t}(30)=2.20, \mathrm{p}<.05)$. Likewise, a higher learning rate in the positive $(\mathrm{M}=0.03, \mathrm{SD}=0.03)$ than in the neutral mood group $(\mathrm{M}=0.00, \mathrm{SD}=0.01)$ was also evidenced following negative feedback $(\mathrm{t}(30)=2.61, \mathrm{p}<.05)$.

The group comparison performed on the inverse-gain parameter $(\beta)$ revealed no significant effect ( $\mathrm{p}>.05$; positive mood group: $\mathrm{M}=0.58, \mathrm{SD}=0.50$; neutral mood group: $\mathrm{M}=0.057$, $\mathrm{SD}=0.018)$

We also computed the Bayesian Information Criterion (BIC) for this model (Model I; see Table 2). We also compared the BIC obtained for this model (allowing different learning rates for positive and negative feedback) to another model (Model III in Table 2) where the learning rate was the same for positive and negative feedback. However, as can be seen from Table 2 we obtained a better fit (i.e., significantly lower BIC) with the former compared to the latter model.

In our model, the values of both the chosen and the unchosen response options were updated after each feedback, in such a way that their values always summed to 1 . So after each choice, the value of the non-selected response increased/decreased with the same amount as the value of the chosen response decreased/increased. We felt is was appropriate to use this 'doubleupdate' model (cf. Matsumoto et al., 2007) as participants were to learn which of the 2 responses was most often associated ("correct") with a given stimulus, and these stimulus-response associations remained stable during the experiment (i.e., evidence in favor of one response automatically also provides evidence against the other response, and v.v.). However, this way of modeling may potentially make the interpretation of positive vs. negative learning rates more difficult. Accordingly, we also computed two alternative 'single-update' models (in which only 
the value of the chosen response was updated; see Models II and IV in Table 2). However, for these two models, the BIC was significantly larger (reflecting a worse model fit) than for our main model.

Ratings. The mixed-model ANOVA carried out on the clarity ratings showed a significant main effect of Condition $\left(F(2,60)=730.3, p<.01, \eta^{2}=1.0\right)$. The analysis performed on the certainty ratings revealed a main effect of Condition alike $\left(F(2,60)=141.5, p<.01, \eta^{2}=.9\right)$. These results showed that perceived clarity and response certainty went down monotonically when S-R probability decreased. However, positive mood did not influence these subjective ratings (p's $>$ .9). Clarity was higher for the deterministic condition $(\mathrm{M}=83.0, \mathrm{SD}=5.0)$ compared to the probabilistic condition $(\mathrm{M}=75.7, \mathrm{SD}=6.7 ; \mathrm{t}(31)=6.8, \mathrm{p}<.01)$, while clarity for the latter was also significantly higher than for the random condition $(\mathrm{M}=37.4, \mathrm{SD}=5.6 ; \mathrm{t}(31)=24.9, \mathrm{p}<.01)$. Likewise, certainty was the highest for the deterministic condition $(\mathrm{M}=65.3, \mathrm{SD}=8.4)$ and intermediate for the probabilistic condition $(\mathrm{M}=48.8 ., \mathrm{SD}=9.0 ; \mathrm{t}(31)=9.5, \mathrm{p}<.01)$. Certainty was also significantly higher in the probabilistic compared to the random condition $(M=36.3, S D=8.5$; $\mathrm{t}(31)=8.5, \mathrm{p}<.01)$.

In contrast, positive mood did reliably affect the subjective experience of participants regarding the amount of positive vs. negative feedback they thought they had received during the experiment, as well as their like-dislike reactions to them (see Table 3). Participants in the positive mood group reported having received significantly more positive than negative feedback, compared to the neutral group $(\mathrm{t}(30)=3.7, \mathrm{p}<.01)$. Moreover, the former participants felt happier than the latter when receiving positive feedback $(\mathrm{t}(30)=3.4, \mathrm{p}<.01)$, while they were also more unhappy when receiving negative feedback when compared to the neutral mood group $(\mathrm{t}(30)=3.9$, $\mathrm{p}<.01)$. 
Questionnaires. Independent t-tests failed to reveal significant group differences for the BDI (Table 3) or resilience scale (all p's $>$.6). The two groups also had a balanced performance regarding the way they filled out the Frederickson's list of emotions (i.e., no significant group differences for the frequencies of the emotional words chosen, all p's >.8).

\section{ERP Results}

The analysis carried out on the ERN mean amplitudes showed a significant main effect of Condition $\left(\mathrm{F}(2,60)=10.8, \mathrm{p}<.01, \eta^{2}=.3\right)$ and Accuracy $\left(\mathrm{F}(1,30)=\mathrm{F}(1,30)=79.6, \mathrm{p}<.01, \eta^{2}=.7\right)$. The two way Condition* Accuracy interaction $\left(F(2,60)=45.2, p<.01, \eta^{2}=.6\right)$ and the three way Group $*$ Condition*Accuracy interaction $\left(F(2,60)=3.8, p<.05, \eta^{2}=.1\right)$ were significant, indicating that positive mood modulated error processing at the response level in a condition and accuracy specific way (see Figures 2, 3 and 5, and Table 4). To disentangle this interaction effect, we computed difference scores for the ERN (incorrect - correct) per condition, and analyzed how this measure varied depending on condition. Importantly, this ANOVA yielded a significant Group x Condition interaction $\left(\mathrm{F}(2,60)=3.8, \mathrm{p}<.05, \eta^{2}=.1\right)$. A direct group comparison showed that the ERN was larger in the positive $(\mathrm{M}=-4.5, \mathrm{SD}=2.0)$ compared to the neutral mood group $(\mathrm{M}=-2.9, \mathrm{SD}=1.9)$ in deterministic condition $(\mathrm{t}(30)=-2.3, \mathrm{p}<.05)$ only. The two groups did not differ from each other in the two other conditions (all ps>.2).

The analysis performed on the mean amplitudes of the FRN yielded a significant main effect of Condition $\left(F(2,60)=13.6, p<.01, \eta^{2}=.3\right)$ and Accuracy $\left(F(1,30)=11.6, p<.01, \eta^{2}=.3\right)$. The interaction between these two factors did not reach significance $(F(2,60)=1.1, p=.34)$. Moreover, positive mood did not reliably influence these effects (all ps >.1). 
Finally, to verify that positive mood influenced the ERN component selectively (while leaving the FRN component unaffected), we run an omnibus ANOVA with ERP Component (either ERN or FRN), Condition and Group as factors. This analysis showed a significant threeway interaction $\left(\mathrm{F}(2,60)=6.6, \mathrm{p}<.01, \eta^{2}=.2\right)$, confirming that positive mood selectively influenced the ERN component in a condition-specific way (i.e., for the deterministic condition).

\section{DISCUSSION}

In this study we put to the test the assumption that the transient experience of positive mood might alter the way positive vs. negative feedback is used to guide behavior during a standard probabilistic learning task. More specifically, we sought to assess whether positive mood might be accompanied by a change in the way positive reinforcers are processed (Eshel \& Roiser, 2010). To this aim, we used a previously validated probabilistic learning task (Eppinger et al., 2008) enabling us to titrate changes in learning both at the behavioral and electrophysiological (ERP) levels. Learning was not only characterized in terms of accuracy and speed, but also using formal learning parameters extracted from a RL model. A reliable and sustained change in positive affect was brought about in one group of participants using a previously validated MIP (Vanlessen et al., 2013). Their behavioral performance and ERP components were compared to another group of participants, for which the mood was kept neutral throughout the task. The results of this study confirm that the current internal state of the participant (here positive mood) can substantially alter the way feedback information is used during probabilistic learning. 
Whereas positive mood did not merely alter the accuracy and speed of learning at the behavioral level (when compared to a control neutral mood condition), a strength of our study was that we characterized changes in RL as a function of this specific mood state using formal learning parameters, besides these standard behavioral measures. Using this procedure, we found that being in a happy mood increased the learning rate, irrespective of the valence of the (preceding) feedback triggering this updating effect. Interestingly, Frank, Moustafa, Haughey, Curran and Hutchinson (2007) previously argued that an increased learning rate could reflect a rapid and enhanced adaptation to changing outcomes, which proved to be an important component of the internalization process at play during learning. Because this enhancement in the learning rate as a function of positive mood was found for both positive and negative feedback, our new results indirectly suggest that positive mood might foster the (rapid or optimal) internalization of the task rule during probabilistic learning, via modulations of both reward (positive/correct feedback) and punishment (negative/incorrect feedback) processing. By contrast the randomness of participants' choices ${ }^{1}$ (reflected in the inverse-gain, or $\beta$, parameter) was not influenced by positive mood. More generally, these results emphasize the added value of modelbased analysis (relative to the standard accuracy or RT data) to reveal subtle group differences related to the encounter and transient experience of positive mood in our study. As a matter of fact, this dissociation suggests that learning might be qualitatively different between the two mood groups, even though a rough quantitative estimate of learning (based on accuracy aggregated across several trials) fails to reveal reliable group differences (see Figure 1).

\footnotetext{
${ }^{1}$ Previous studies have linked this parameter (beta) to exploration (Jepma \& Nieuwenhuis, 2011). However, the current experimental paradigm (a two-alternative forced-choice task with stable S-R probabilities) was not designed to measure the exploration-exploitation tradeoff. Hence, in the current study this parameter mainly reflects the randomness of participants' choices.
} 
Positive mood was also associated with a larger ERN effect than neutral mood, for the deterministic condition selectively. Classically, the ERN component is thought to reflect prediction error based on the processing of internal (perhaps motor-related) cues (Falkenstein, Hoormann, Christ, \& Hohnsbein, 2000), as opposed to external ones in the case of the FRN (Eppinger et al. 2008). Accordingly, our ERN results are compatible with the notion of a larger RL signal present at the response level (Holroyd \& Coles, 2002), for this specific condition (deterministic) and group (positive mood). With this scenario, positive mood would therefore heighten prediction error encoding (likely within the dorsal ACC and interconnected midbrain dopaminergic brain structures; see Holroyd \& Coles, 2002), if and only if reward probability (acquired through learning) is actually high. These results suggest therefore that positive mood does not simply create an unspecific shift in motivation, or a uniform boost in reward processing (across all conditions in an undifferentiated manner). Instead, the significant interaction effect between mood/group and condition found at the level of the ERN is compatible with the idea that positive mood may boost reward prediction error during learning when the probability of getting a reward (as computed based on an internal/motor representation - ERN component) is high.

Interestingly, earlier ERP studies consistently found a larger ERN component in participants characterized by internalizing traits (sub clinical) or disorders (clinical), or negative affect broadly defined (Vaidyanathan, Nelsonm \& Patrick, 2012; Olvet \& Hajcak, 2008; Aarts \& Pourtois 2010; Moser, Moran, Schroder, Donnellan, \& Yeung, 2013). By comparison, our new electrophysiological results show that the transient experience of positive affect can be associated with an augmented ERN effect alike, casting doubt on the idea that an overactive ERN necessarily reflects a stable endophenotype for internalizing traits or disorders along the main psychopathology continuum (Olvet \& Hajcak, 2008). Therefore, this neurophysiological effect 
may reflect a transient change and increase following the induction of positive mood in the dopaminergic-dependent neural system (connecting the basal ganglia to the rostral cingulate zone) thought to underlie reward prediction error and in turn the generation of the ERN component (see Holroyd \& Coles, 2002). Noteworthy, this conjecture was already made by Ashby et al. (1999) about 15 years ago, but never or rarely put to the test directly. This theory assumes that during periods of mild positive affect, there is a concomitant increased dopamine release in the mesocorticolimbic system, and also the nigrostriatal system, which can in turn influences performance on a variety of cognitive tasks. Accordingly, our new neurophysiological results are important because they lend empirical support to this dominant model, while they also show that the ERN component is not only sensitive to negative affect or punishment (trait characteristics), but also changes in positive mood (state effect).

It should be noted however that this somewhat subtle change in the way positive feedback (or reward) is processed after the induction of positive mood did not seem to be related to (or explained by) obvious modifications in the way participants actually perceived and experienced “retrospectively" this probabilistic learning task. In both groups, participants' subjective visibility and certainty of the S-R associations varied according to the experimental conditions in the predicted direction. Notwithstanding a lack of group difference for these two variables, an interesting finding was that participants in the positive mood group reported to have received more positive feedback than in the neutral mood group, as well as to like them more, even though we could not assess whether this effect was condition specific. Future studies are needed to test whether these learning (rate) and neurophysiological effects (ERN and FRN components) might predict reliable changes in the way probabilistic learning is eventually experienced by the participants (after the induction of positive mood). 
For the FRN component, we found that this feedback-locked ERP activity showed a clear amplitude modulation depending on the varying reward probabilities (i.e., the FRN component was large for the random and probabilistic conditions, but small for the deterministic condition; see also Eppinger et al., 2008), irrespective of the mood change however. Hence, positive mood appears to exert selective influences on reward prediction error during probabilistic learning, namely at the motor (internal) level, while leaving the feedback (external) level unchanged. Although these ERP results reveal some specificity in the way positive mood may shape reward processing during learning, we note nonetheless that the results obtained for the learning parameters (RL) point to a general increase in learning rate following positive feedback (after the induction of positive mood). Moreover, we did not find any significant correlation between amplitude variations at the level of the ERN and this learning parameter (nor for the inverse-gain parameter). This discrepancy between the ERP and computational modeling results might stem from methodological reasons (e.g., averaging of several trials regardless of trial history in the case of FRN vs. updating of beliefs depending on the most recent feedback in the case of learning rate).

A few limitations have to be noted. As is often the case with research on positive mood, it remains difficult to disentangle effects of positive valence from arousal per se. Our results clearly show that participants in the positive mood group not only experienced more happiness and pleasantness relative to the neutral mood group, but also more arousal (at a subjective level though). However, earlier studies found that valence, but not arousal, did contribute to adaptive goal-directed behavior in front of conflicting situations or events (van Steenbergen, Band, \& Hommel, 2010). On the other hand, arousal per se appears unlikely to account for the complex interaction effects on the FRN and ERN components in our study. Another caveat concerns the 

as well as ERN component on the one hand, but on the other hand, the lack of obvious group differences regarding response accuracy during the probabilistic learning task. Three elements are here noteworthy. First, accuracy data were computed in line with Eppinger et al. (2008), where bins (i.e., average of performance across several trials) were eventually used. Although this method is probably suited to grasp "gross" changes in learning depending on time and condition (see Figure 1), it is probably not sensitive enough to detect finer alterations occurring on a trial by trial basis during probabilistic learning, as a function of positive mood, as our modeling and ERP data reveal. Second, our results also show that participants did not perform at ceiling at the end of the experimental session. It is possible that group differences on accuracy would appear when using more trials. Third, the absence of any group difference regarding accuracy (bearing also in mind that participants in the two groups showed normal learning curves depending on the experimental condition; see Figure 1) was actually an asset in the present case, because we could then compare learning parameters and ERP data when the amount of response errors (and hence negative feedback) was actually balanced between the two groups. In other words, the present results cannot be explained by a general asymmetry or imbalance in accuracy during the probabilistic learning task across the two groups.

To conclude, the results of this study provide evidence for a selective modulatory effect exerted by positive mood on probabilistic learning. At the behavioral level, positive mood heightens the learning rate (as opposed to merely increasing "exploration" for example), in particular when positive feedback can be used to guide learning directly. At the electrophysiological level, this effect is primarily expressed in the way (reward) prediction error is processed (at the response levels) when the certainty and visibility regarding the S-R 
associations are (relatively) high (i.e., in the deterministic condition). An open question for future research concerns the potential costs and benefits for the organism associated with this change in reward prediction error following the induction of positive mood. For example, whether or not an enhanced or sharper reward prediction error mechanism during the encounter or experience of positive mood may foster learning (compared to a neutral or negative mood), remains to be established at the empirical level. In this study, we failed to observe improvements in learning at the behavioral level following the induction of positive mood, although positive mood influenced the learning rate and shaped a well-known electrophysiological marker of reward prediction error (i.e., the ERN).

\section{ACKNOWLEDGEMENTS}

This worked is supported by a Concerted Research Action Grant from Ghent University awarded to RDR (\#BOF10/GOA/014) and by the Belgian Science Policy, Interuniversity Attraction Poles program (P7/11). GP is funded by the European Research Council (Starting Grant \#200758) and Ghent University (BOF Grant \#05Z01708). The authors have no conflict of interest to declare.

\section{REFERENCES}

Aarts, K., \& Pourtois, G. (2010). Anxiety not only increases, but also alters early errormonitoring functions. Cognitive, Affective \& Behavioral Neuroscience, 10(4), 47992. doi:10.3758/CABN.10.4.479. 
Aarts, K., \& Pourtois, G. (2012). Anxiety disrupts the evaluative component of performance monitoring: An ERP study. Neuropsychologia, 50, 1286- 1296.

Aarts, K., De Houwer, J., \& Pourtois, G. (2013). Erroneous and correct actions have a different affective valence: Evidence from ERPs. Emotion, 13(5):960-73.

Ashby, F.G., Isen, A.M., \& Turken, U. (1999). A neuropsychological theory of positive affect and its influence in cognition. Psychological Review, 106 (3), 529-550.

Beck, A.T., Steer, R.A., Ball, R., \& Ranieri, W. (1996). Comparison of Beck Depression Inventories- IA and II in psychiatric outpatients. Journal of Personality Assessment, 67(3), 588-597.

Bediou, B., Koban, L., Rosset, S., Pourtois, G., \& Sander, D. (2012). Delayed monitoring of accuracy errors compared to commission errors in ACC. NeuroImage, 60(4), 192536. doi:10.1016/j.neuroimage.2012.02.010.

Behrens, T. E. J., Woolrich, M. W., Walton, M. E., \& Rushworth, M. F. S. (2007). Learning the value of information in an uncertain world. Nature Neuroscience, 10(9), 121421. doi:10.1038/nn1954.

Bradley, B., \& Mathews, A. (1983). Negative self-schemata in clinical depression. The British Journal of Clinical Psychology / the British Psychological Society, 22 (Pt 3), 173-181. doi:10.1111/j.2044-8260.1983.tb00598.x. 
Carbonnell, L., \& Falkenstein, M. (2006). Does the error negativity reflect the degree of response conflict? Brain research, 1095(1), 124-130. doi:10.1016/j.brainres.2006.04.004.

Coleman, T. F., \& Li, Y. (1996). An Interior Trust Region Approach for Nonlinear Minimization Subject to Bounds. SIAM Journal on Optimization. doi:10.1137/0806023.

Daw, N.D., O’Doherty, J.P., Dayan, P., Seymour, B., \& Dolan, R.J. (2006). Cortical substrates for exploratory decisions in humans. Nature, 441, 876-879. doi: 10.1038/nature04766.

Eppinger, B., Kray, J., Mock, B., \& Mecklinger, A. (2008). Better or worse than expected? Aging, learning, and the ERN. Neuropsychologia, 46(2), 521-539.

Eshel, N., \& Roiser, J.P. (2010). Reward and punishment processing in depression. Biological Psychiatry, 68, 188-124.

Falkenstein, M., Hohnsbein, J., Hoormann, J., \& Blanke, L. (1991). Effects of crossmodal divided attention on late ERP components. II. Error processing in choice reaction tasks. Electroencephalography and Clinical Neurophysiology, 78, 447-455. doi:10.1016/0013-4694(91)90062-9.

Falkenstein, M., Hoormann, J., Christ, S., \& Hohnsbein, J. (2000). ERP components on reaction errors and their functional significance: a tutorial. Biological Psychology, 51(2-3), 87-107. doi:10.1016/S0301-0511(99)00031-9. 
Frank, M. J., Moustafa, A. A., Haughey, H. M., Curran, T., \& Hutchison, K. E. (2007). Genetic triple dissociation reveals multiple roles for dopamine in reinforcement learning. Proceedings of the National Academy of Sciences of the United States of America, 104(41), 16311-16316.

Frank, M. J., Woroch, B.S., \& Curran, T. (2005). Error-related negativity predicts reinforcement learning and conflict biases. Neuron, 47, 495-501.doi: 10.1016/j.neuron.2005.06.020.

Fredrickson, B.L. (2001). The role of positive emotions in positive psychology. The broaden-and-build-theory of positive emotions. American Psychologist, 56(3), 218226.

Fredrickson, B.L. (2004). The broaden-and-build theory of positive emotions. Philos Trans R Soc Lond B Biol Sci, 359(1449), 1367-1378. doi:10.1098/rstb.2004.1512.

Garland, E. L., Fredrickson, B., Kring, A.M., Johnson, D.P., Meyer, P.S., \& Penn, D.L. (2010). Upward spirals of positive emotions counter downward spirals of negativity: Insights from the broaden-and-build theory and affective neuroscience on the treatment of emotion dysfunctions and deficits in psychopathology. Clinical Psychology Review, 30, 849-864.

Gehring, W. J., Goss, B., Coles, M. G. H., Meyer, D. E., \& Donchin, E. (1993). A Neural System for Error-Detection and Compensation. Psychological Science, 4(6), 385390. Retrieved from <Go to ISI >://A1993MF61300008. 
Gehring, W. J., \& Willoughby, A. R. (2002). The medial frontal cortex and the rapid processing of monetary gains and losses. Science (New York, N.Y.), 295(5563), 2279-2282. doi:10.1126/science.1066893.

Gentsch, A., Ullsperger, P., \& Ullsperger, M. (2009). Dissociable medial frontal negativities from a common monitoring system for self- and externally caused failure of goal $\begin{array}{lll}\text { achievement. } & \text { NeuroImage, } & \text { 2023-2030. }\end{array}$ doi:10.1016/j.neuroimage.2009.05.064.

Gratton, G., Coles, M. G., \& Donchin, E. (1983). A new method for off-line removal of ocular artifact. Electroencephalography \& Clinical Neurophysiology, 55, 468-484.

Hills, T.T., Todd, P.M., \& Goldstone, R.L. (2010). The central executive as a search process: priming exploration and exploitation across domains. J Exp Psychol Gen., 139(4), 590-609. doi: 10.1037/a0020666.

Holmes, E.A., Coughtrey, A.E., \& Connor, A. (2008). Looking at or through rose-tinted glasses? Imagery perspective and positive mood. Emotion, 8(6), 875-879.

Holmes, E.A., Mathews, A., Dalgleish, T., \& Mackintosch, B. (2006). Positive interpretation training: Effects of mental imagery versus verbal training on positive mood. Behavioral Therapy, 37(3), 237-247.

Holroyd, C. B., \& Coles, M. G. (2002). The neural basis of human error processing: Reinforcement learning, dopamine, and the error-related negativity. Psychological Review, 109(4), 679-709. 
Jepma, M., \& Nieuwenhuis, S. (2011). Pupil diameter predicts changes in the explorationexploitation trade-off : Evidence for the adaptive gain theory. Journal of Cognitive Neuroscience, 23(7), 1587-96. doi: 10.1162/jocn.2010.21548.

Lange, S., Leue, A., \& Beauducel, A. (2012). Behavioral approach and reward processing: Results on feedback-related negativity and P3 component. Biological Psychology, $89(2), 416-425$.

Matsumoto, M., Matsumoto, K., Abe, H., \& Tanaka, K. (2007). Medial prefrontal cell activity signaling prediction errors of action values. Nat Neurosci., 10(5):647-56.

Mies, G.W., van der Veen, F.M., Tulen, J.H.M., Birkenhäger, T.K., Hengeveld, M.W., \& van der Molen, M.W. (2011). Drug-free patients with major depression show an increased electrophysiological response to valid and invalid feedback. Psychological Medicine 41, 2515-2525. doi:10.1017/S0033291711000778.

Miltner, W. H., Braun, C. H., \& Coles, M. G. (1997). Event-related brain potentials following incorrect feedback in a time-estimation task: Evidence for a "generic" neural system for error detection. Journal of cognitive neuroscience, 9(6), 788-798.

Moser, J. S., Moran, T. P., Schroder, H. S., Donnellan, M. B., \& Yeung, N. (2013). On the relationship between anxiety and error monitoring: a meta-analysis and conceptual framework. Frontiers in Human Neuroscience, 7(August), 466. doi:10.3389/fnhum.2013.00466.

Nadler, R. T., Rabi, R., \& Minda, J. P. (2010). Better mood and better performance. Learning rule-described categories is enhanced by positive mood. Psychological 
Science $\square$ : A Journal of the American Psychological Society / APS, 21(12), 1770 1776. doi:10.1177/0956797610387441

Nieuwenhuis, S., Slagter, H. A., von Geusau, N. J. A., Heslenfeld, D. J., \& Holroyd, C. B. (2005). Knowing good from bad: differential activation of human cortical areas by positive and negative outcomes. The European Journal of Neuroscience, 21(11), 3161-3168. doi:10.1111/j.1460-9568.2005.04152.x.

Olvet, D. M., \& Hajcak, G. (2008). The error-related negativity (ERN) and psychopathology: toward an endophenotype. Clin Psychol Rev, 28(8), 1343-1354. doi:10.1016/j.cpr.2008.07.003.

Picton, T.W., Bentin, S., Berg, P., Donchin, E., Hillyard, S.A., Johnson, R., ..., Taylor, M.J. (2000). Guidelines for using human event-related potentials to study cognition: Recording standards and publication criteria. Psychophysiology, 37,127-152.

Portzky, M., Wagnild, G., De Bacquer D., \& Audenaert, K. (2010). Psychometric evaluation of the Dutch Resilience Scale RS-nl on 3265 healthy participants: A confirmation of the association between age and resilience found with the Swedish version. Scandinavian Journal of Caring Sciences, 1, 86-92. doi:10.1111/j.14716712.2010.00841.x.

Pourtois, G. (2011). Early Error Detection Predicted by Reduced Pre-response Control Process: An ERP Topographic Mapping Study. Brain Topography, 23(4),403-22. 
Rossion, B., \& Pourtois, G. (2004). Revisiting Snodgrass and Vanderwart's object pictorial set: The role of surface detail in basic-level object recognition. Perception, 33(2), 217-236.

Sallet, J., Camille, N., \& Procyk, E. (2013). Modulation of feedback-related negativity during trial-and-error exploration and encoding of behavioral shifts. Frontiers in Neuroscience, 7, 209. doi:10.3389/fnins.2013.00209.

Sutton, R., \& Barto, A. G. (1998). Reinforcement learning: an introduction. Cambridge, MA: MIT Press.

Vaidyanathan, U., Nelson, L. D., \& Patrick, C. J. (2012). Clarifying domains of internalizing psychopathology using neurophysiology. Psychological Medicine. doi:10.1017/S0033291711001528.

Van Steenbergen, H., Band, G. P., \& Hommel, B. (2010). In the mood for adaptation: how affect regulates conflict-driven control. Psychological Science, 21(11), 1629-1634. doi:10.1177/0956797610385951.

Vanlessen, N., Rossi, V., De Raedt, R., \& Pourtois, G. (2013). Positive emotion broadens attention focus through decreased position-specific spatial encoding in early visual cortex: Evidence from ERPs. Cognitive, Affective \& Behavioral Neuroscience, 13(1), 60-79. doi: 10.3758/s13415-012-0130-x.

Vanlessen, N., Rossi, V., De Raedt, R., \& Pourtois, G. (2014). Feeling happy enhances early spatial encoding of peripheral information automatically: electrophysiological time- 
course and neural sources. Cognitive Affective and Behavioral Neuroscience,14 (3), 951-969. doi: 10.3758/s13415-014-0262-2.

Walsh, M. M., \& Anderson, J. R. (2011). Modulation of the feedback-related negativity by instruction and experience. Proceedings of the National Academy of Sciences of the United States of America, 108(47), 19048-19053. doi:10.1073/pnas.1117189108

Walsh, M. M., \& Anderson, J.R. (2012). Learning from experience: Event-related potential correlates of rewards processing, neural adaptation, and behavioral choice. Neuroscience and Behavioral Reviews, 36, 1870-1884.

Walsh, M.M., \& Anderson, J.R. (2014). Navigating complex decision spaces: Problems and paradigms in sequential choice. Psychological Bulletin, 140(2):466-86. doi: $10.1037 / \mathrm{a} 0033455$

Watkins, E.R., \& Moberly, N.J. (2009). Concreteness training reduces dysphoria: A pilot proof-of-principle-study. Behavioral Research and Therapy, 47(1), 48-53. doi: 10.1016/j.brat.2008.10.014.

\section{FIGURES LEGEND}

Figure 1. Accuracy data (i.e., proportion of correct responses) decomposed as a function of bin, condition and group. 
Figure 2. Mean amplitude in $\mu \mathrm{V}$ (+ 1 S.E.M) of the difference score (incorrect minus correct) for the ERN component at the electrode FCz, shown separately for each group and each condition.

Figure 3. Grand average ERP waveforms for the response-locked ERP data (electrode FCZ), separately for each group, condition and accuracy level.

Figure 4. Grand average ERP waveforms for the feedback-locked ERP data (electrode FCZ), separately for each group, condition and accuracy level.

Figure 5. Topographical map (horizontal view) of the ERN and FRN effects, separately for each group and condition. 
Table 1. Results of the MIP. Means (+1 Standard Deviation) and results of the group comparison (based on independent-samples t-tests) between the Positive $(n=16)$ and the Neutral $(n=16)$ mood group.

\begin{tabular}{|c|c|c|c|c|c|c|c|c|c|c|c|c|}
\hline \multirow{2}{*}{$\begin{array}{c}\text { Measure } \\
\text { point }\end{array}$} & \multicolumn{3}{|c|}{ Happiness } & \multicolumn{3}{|c|}{ Pleasantness } & \multicolumn{3}{|c|}{ Sadness } & \multicolumn{3}{|c|}{ Arousal } \\
\hline & Positive & Neutral & t-test & Positive & Neutral & t-test & Positive & Neutral & t-test & Positive & Neutral & t-test \\
\hline Baseline & $39.9(16.9)$ & $44.3(17.7)$ & -0.7 & $44.7(14.3)$ & $39.4(22.1)$ & 0.8 & $11.2(15.0)$ & $8.8(11.2)$ & 0.5 & $5.5(2.2)$ & $5.0(2.7)$ & 0.6 \\
\hline 1 & $59.6(14.8)$ & $43.7(19.2)$ & $2.6^{*}$ & $58.3(5.8)$ & $37.2(21.6)$ & $3.1 * *$ & $7.7(12.0)$ & $8.3(8.3)$ & -0.2 & $4.2(2.0)$ & $5.9(2.4)$ & $-2.2 *$ \\
\hline 2 & $61.0(13.3)$ & $36.4(22.8)$ & $3.7 * *$ & $62.9(13.1)$ & 40.7 (23.7) & $3.3 * *$ & $6.9(6.1)$ & $7.6(10.0)$ & -0.3 & $3.5(1.2)$ & $6.0(2.0)$ & $-4.3 *$ \\
\hline 3 & 58.7 (13.9) & $35.7(25.5)$ & $3.2 * *$ & $59.3(16.0)$ & $36.9(26.7)$ & $2.9 * *$ & $8.4(14.5)$ & $4.1(8.5)$ & 1.0 & $4.1(1.7)$ & $5.1(2.9)$ & -1.2 \\
\hline 4 & $61.8(15.0)$ & $36.9(27.8)$ & $3.2 * *$ & $63.6(14.8)$ & $38.0(29.1)$ & $3.4 * *$ & $6.6(9.8)$ & $5.7(12.5)$ & 0.2 & $3.7(1.7)$ & $5.9(2.7)$ & $-2.8 * *$ \\
\hline 5 & $63.9(13.4)$ & $32.8(31.0)$ & $3.7 * *$ & $64.1(15.9)$ & $32.8(33.3)$ & $3.4 * *$ & $6.9(9.3)$ & $5.8(16.9)$ & 0.2 & $3.4(1.5)$ & $5.4(2.9)$ & $-2.4 *$ \\
\hline 6 & 63.7 (13.7) & $42.9(25.3)$ & $2.9 * *$ & $63.3(16.3)$ & $42.0(26.2)$ & $2.8 * *$ & $11.9(22.8)$ & $7.2(8.2)$ & 0.8 & $4.3(1.5)$ & $5.7(2.6)$ & -2.0 \\
\hline
\end{tabular}

$* \mathrm{p}<.05, * * \mathrm{p}<.01$ 
Table 2. BIC (mean +1 Standard Deviation in parenthesis) obtained for the four different models, separately (see text for details). Model I: Separate Learning Rates for Positive and Negative feedback, and inter-dependent response alternatives; Model II: Separate Learning Rates for Positive and Negative feedback, and independent response alternatives; Model III: One Learning Rate for Positive and Negative feedback, and inter-dependent response alternatives; Model II: One Learning Rate for Positive and Negative feedback, and independent response alternatives.

\begin{tabular}{llll}
\hline & BIC & Pairwise comparisons & t-test $(\mathrm{df}=31)$ \\
\hline Model I & $712.26(119.86)$ & Model I vs. Model II & $-4.78^{* *}$ \\
Model II & $721.68(115.68)$ & Model I vs. Model III & $-8.50^{* *}$ \\
& & & \\
Model III & $773.43 .(94.80)$ & Model I vs. Model IV & $-8.35^{* *}$ \\
& & & \\
Model IV & $774.96(95.78)$ & Model II vs. Model III & -0.72 \\
\hline
\end{tabular}

$* \mathrm{p}<.05, * * \mathrm{p}<.01$ 
Table 3. Subjective ratings (regarding the number of positive feedback received throughout the experiment, as well as the like-dislike reaction to them) and BDI scores. For each measure, the mean (+ 1 Standard Deviation in parenthesis), as well as the direct group comparison (using unpaired t-test) are provided.

\begin{tabular}{|c|c|c|c|c|c|c|c|c|c|c|c|}
\hline \multicolumn{3}{|c|}{ Estimate of positive feedback received } & \multicolumn{3}{|c|}{ Liking positive feedback } & \multicolumn{3}{|c|}{ Disliking negative feedback } & \multicolumn{3}{|c|}{ BDI } \\
\hline Positive & Neutral & t-test & Positive & Neutral & t-test & Positive & Neutral & t-test & Positive & Neutral & t-test \\
\hline $45.4(9.7)$ & $30.2(13.6)$ & $3.7 * *$ & $47.6(8.7)$ & $36.8(9.2)$ & $3.4 * *$ & $50.7(7.7)$ & $40.1(7.8)$ & $3.9 * *$ & $7.8(7.0)$ & $6.1(5.7)$ & 0.7 \\
\hline
\end{tabular}

$* \mathrm{p}<.05, * * \mathrm{p}<.01$ 
Table 4. Mean ERP activity (1 standard deviation) for each condition and accuracy level, separately for each ERP component and group. Results of the direct pairwise comparisons (degrees of freedom: 15) between the two accuracy levels (correct vs. incorrect), using posthoc t-tests.

\begin{tabular}{|c|c|c|c|c|c|c|c|}
\hline \\
\hline \multirow{2}{*}{ component } & \multirow[t]{2}{*}{ Condition } & \multicolumn{6}{|c|}{ Mood } \\
\hline & & & Positive & & & Neutral & \\
\hline \multirow[t]{4}{*}{ ERN } & & Correct & Incorrect & t-test & Correct & Incorrect & t-test \\
\hline & Deterministic & $-0.9(2.1)$ & $-5.9(2.5)$ & $6.8^{* *}$ & $-0.1(2.0)$ & $-3.4(2.8)$ & $5.9 * *$ \\
\hline & Probabilistic & $-1.5(2.1)$ & $-2.4(1.2)$ & $2.5^{*}$ & $-0.2(2.5)$ & $-1.3(2.7)$ & $3.2 *$ \\
\hline & Random & $-2.2(2.0)$ & $-2.1(1.6)$ & -0.6 & $-0.6(2.2)$ & $-0.9(2.3)$ & 1.0 \\
\hline \multicolumn{8}{|l|}{ FRN } \\
\hline & Deterministic & $-0.4(2.0)$ & $-0.1(4.2)$ & -0.4 & $-0.9(2.1)$ & $-2.6(2.2)$ & $2.7^{*}$ \\
\hline & Probabilistic & $0.7(3.2)$ & $-0.5(3.2)$ & $4.3 * *$ & $-0.2(2.2)$ & $-1.1(2.6)$ & $2.1^{*}$ \\
\hline & Random & $1.7(3.3)$ & $0.2(3.7)$ & $3.2^{* *}$ & $0.2(1.7)$ & $-1.1(2.0)$ & $2.8 * *$ \\
\hline
\end{tabular}

$* \mathrm{p}<.05, * * \mathrm{p}<.01$ 

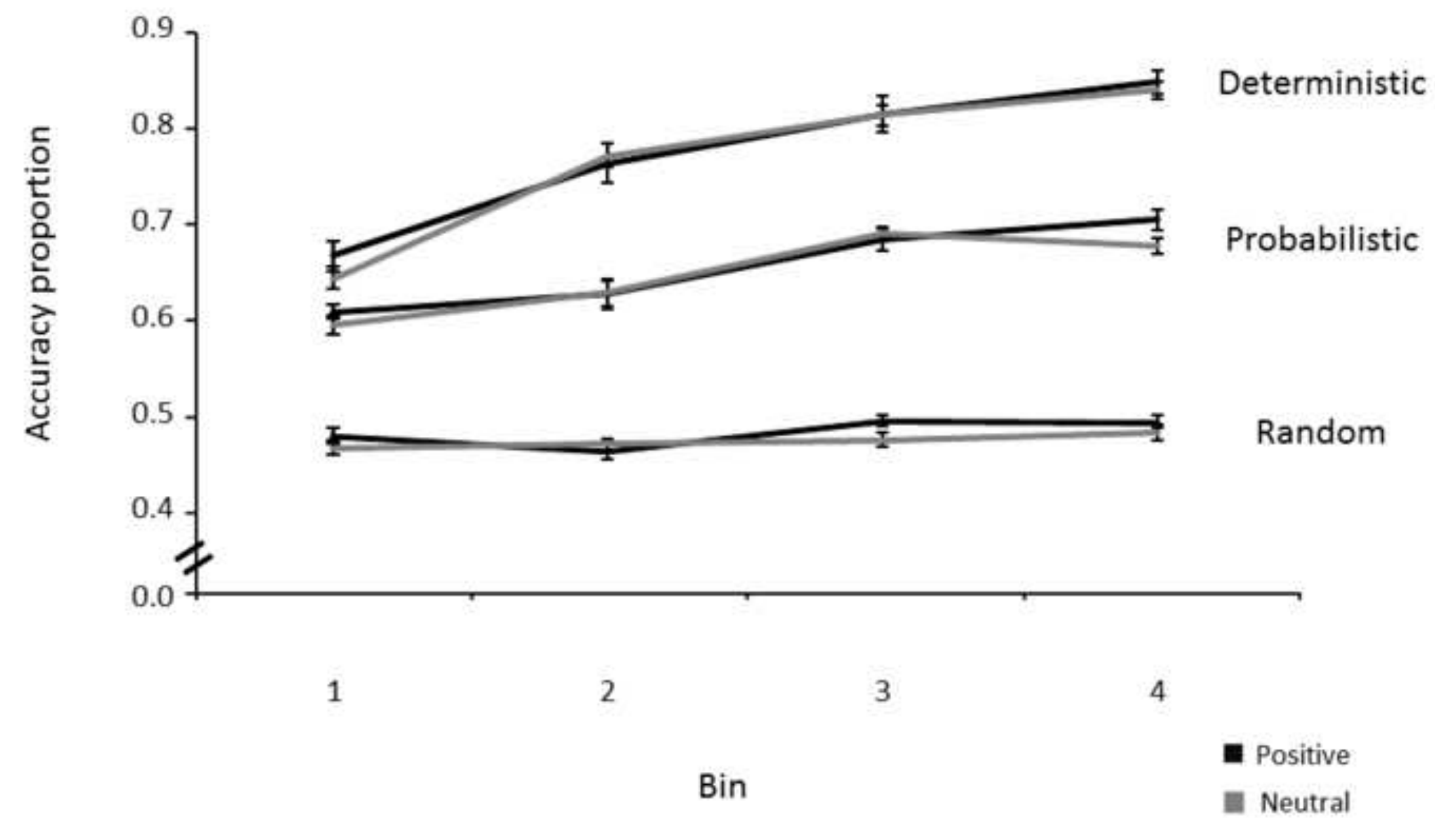


\section{ERN (FCZ electrode)}

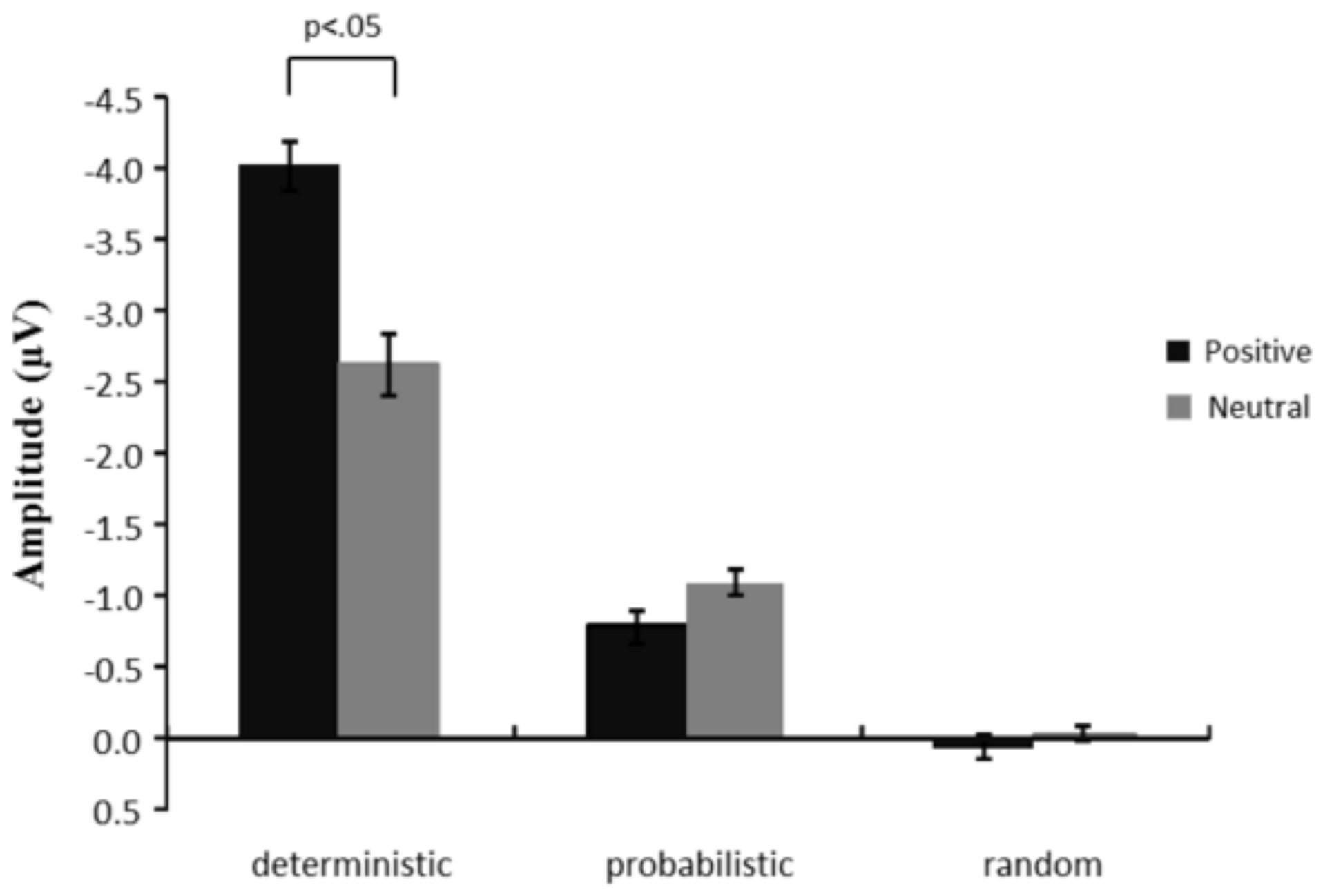




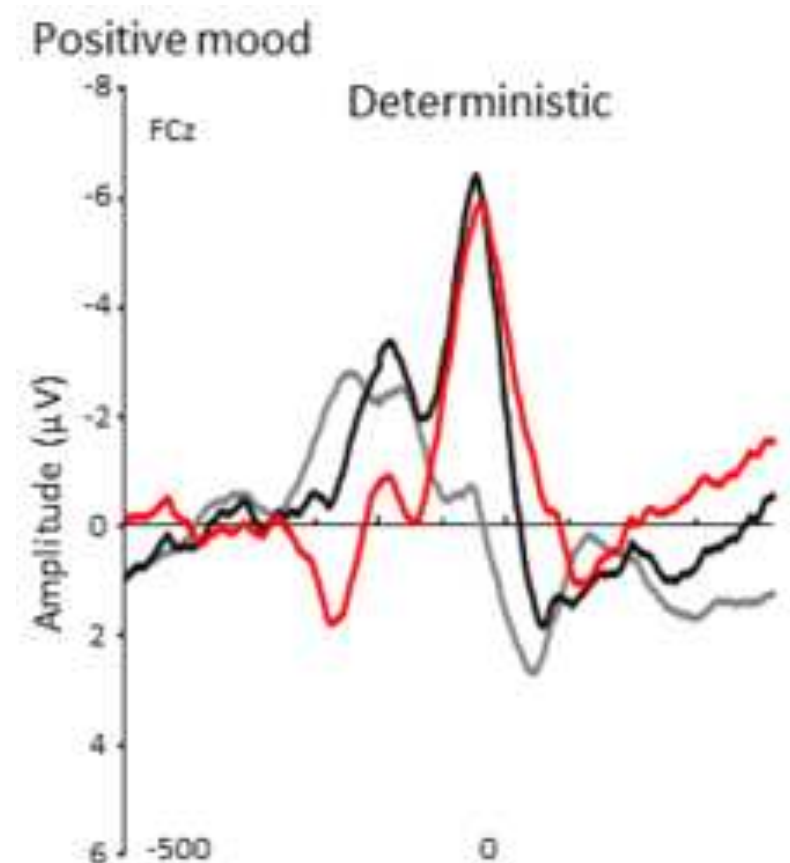

Neutral mood

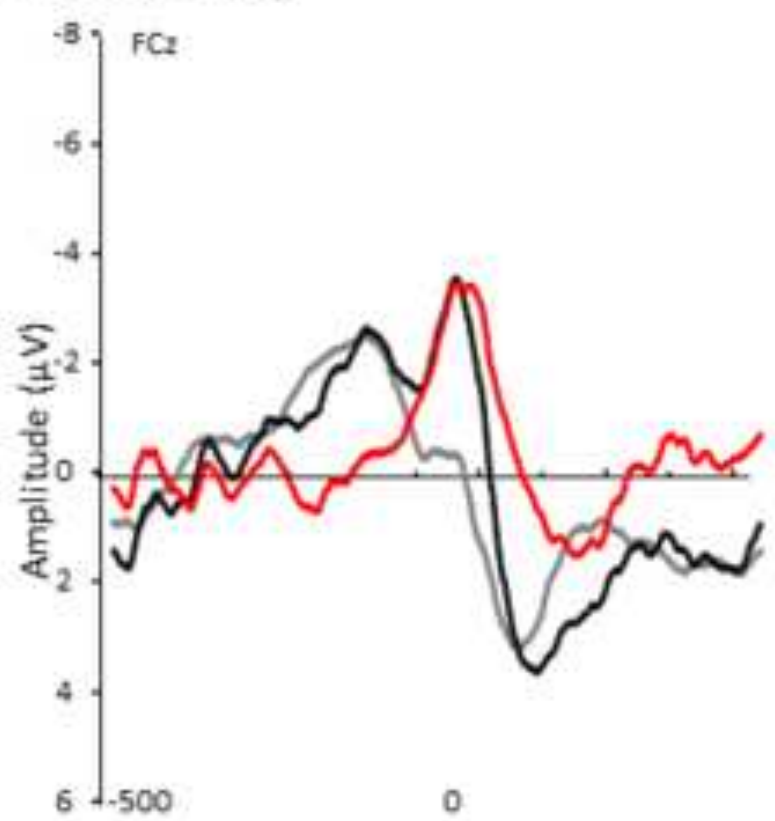

Probabilistic

Random

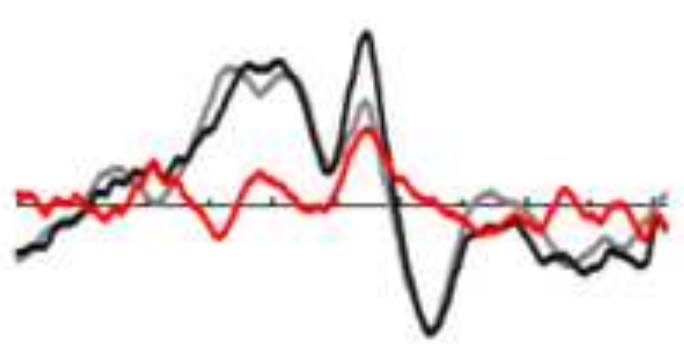

$-500$

0

Time (ms)

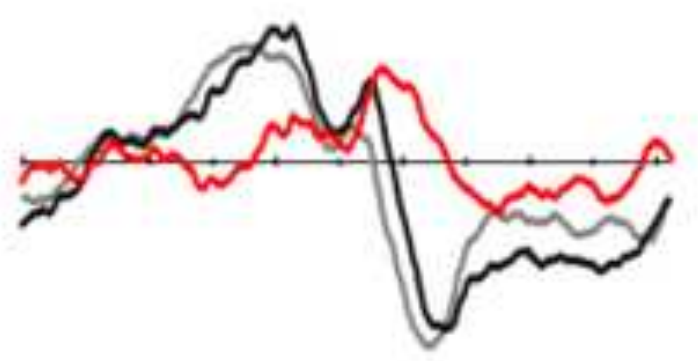

$-500$

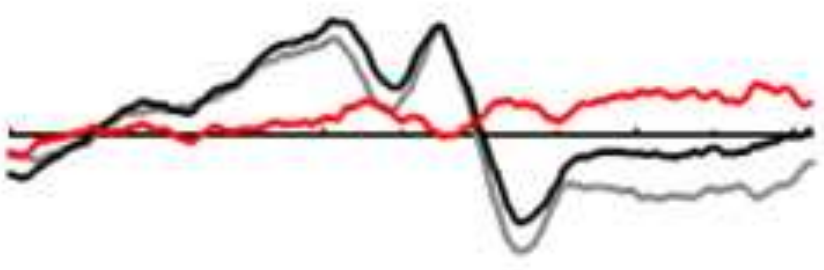

$-500$

Time (ms)

- Incorrect

- Correct

- Difference wave

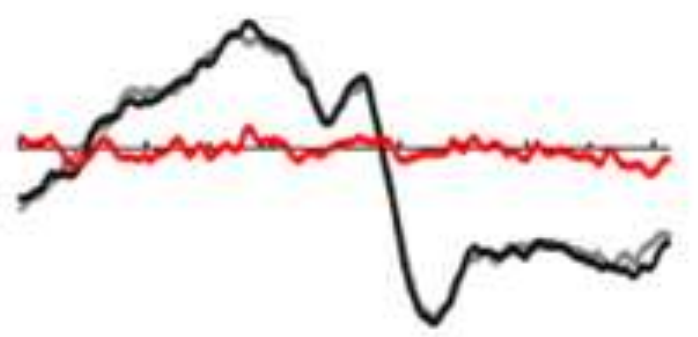

$-500$

0 
Positive mood

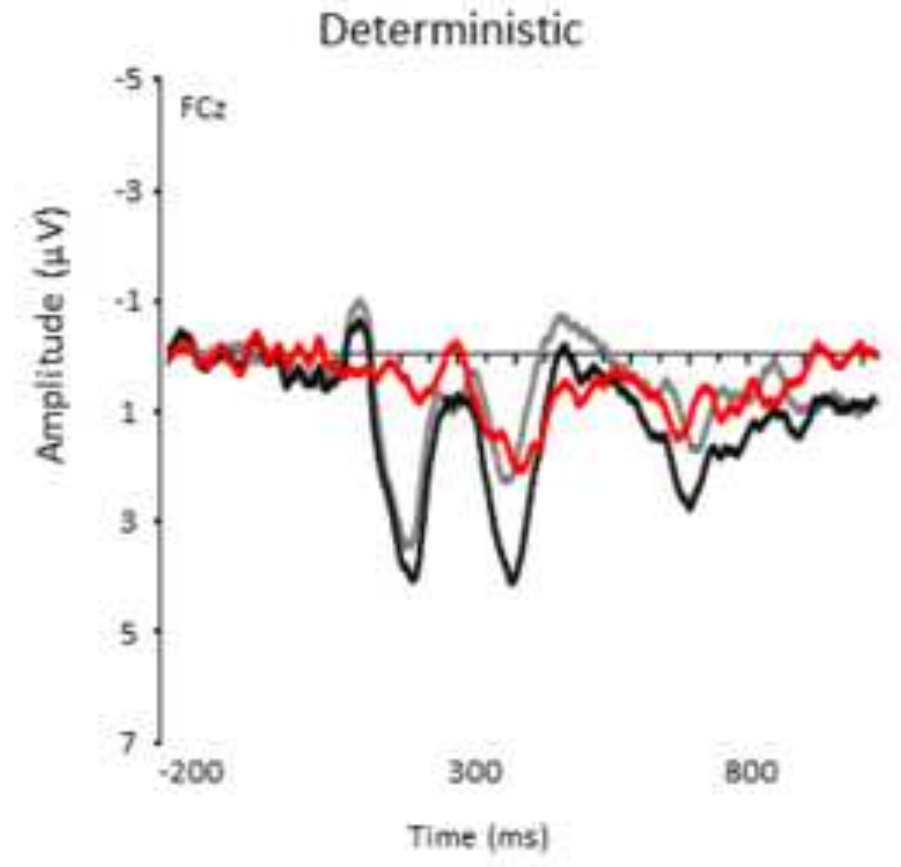

Neutral mood

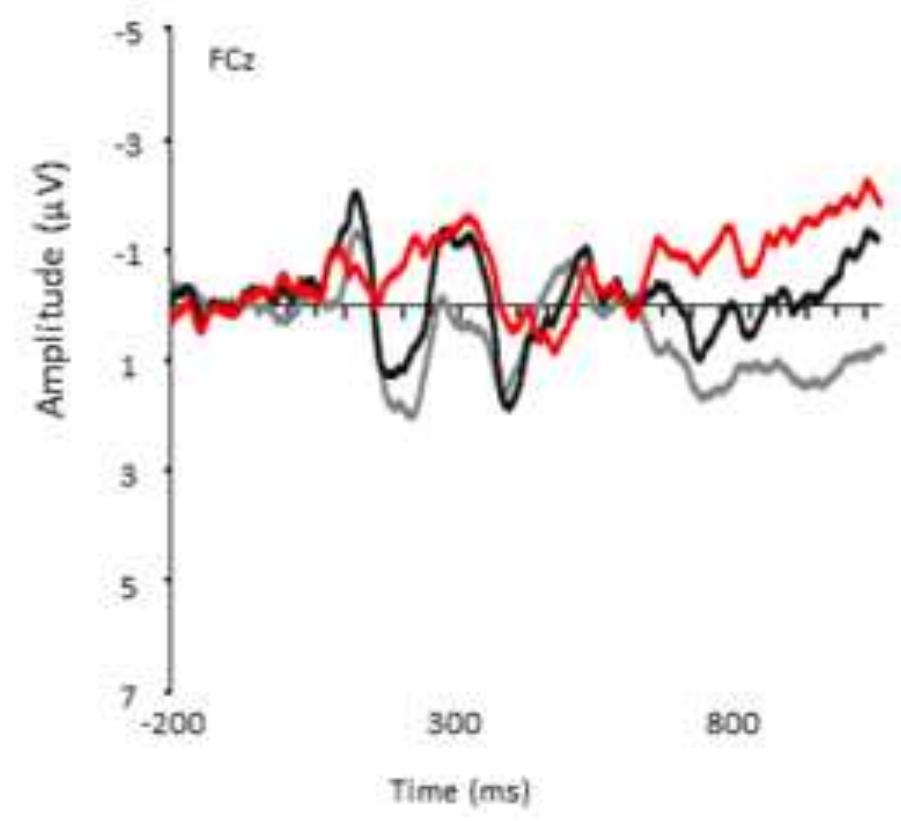

Probabilistic

Random
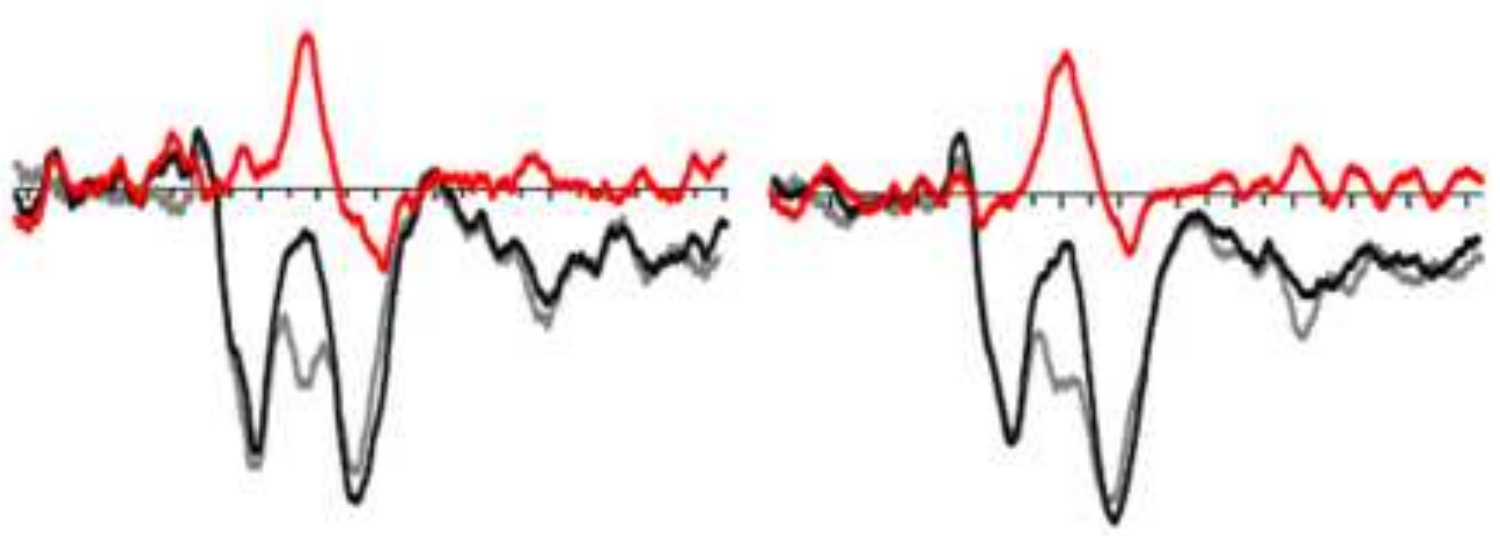

$-200$

300

800

$-200$

300

800

Time (ms)

Time (ms)

- Incorrect

- Correct

- Difference wave
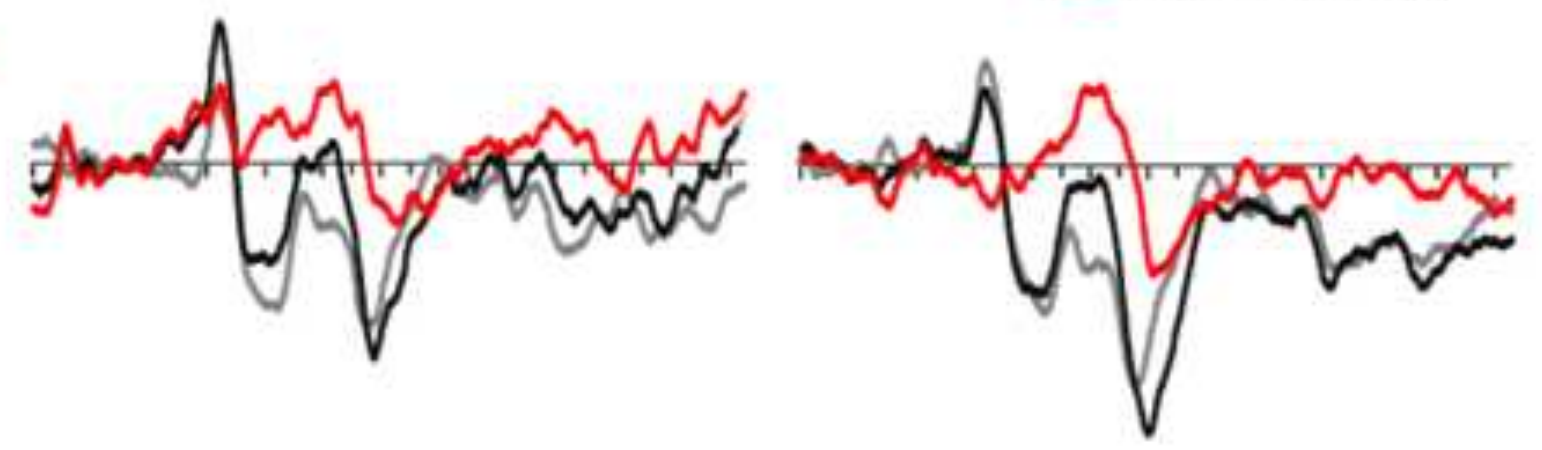

$-200$

300

800

$-200$

300

800 


\section{Deterministic}

Positive mood
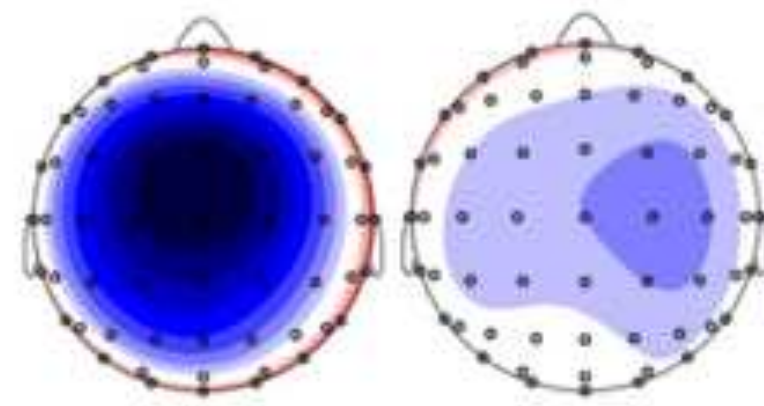

ERN
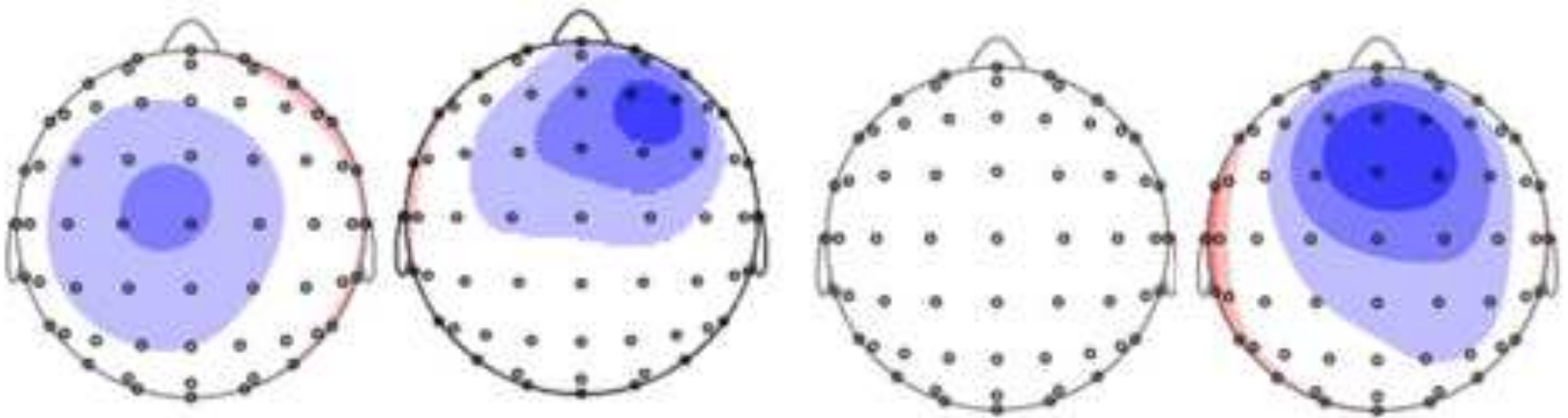

ERN

FRN

ERN

FRN

\section{Neutral mood}

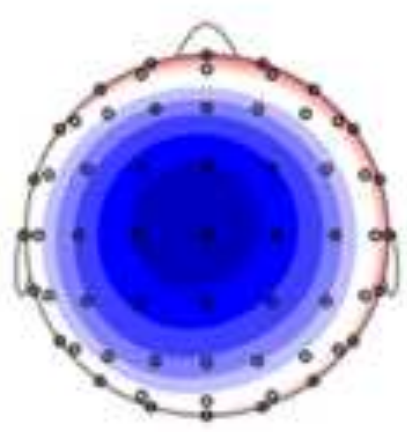

ERN

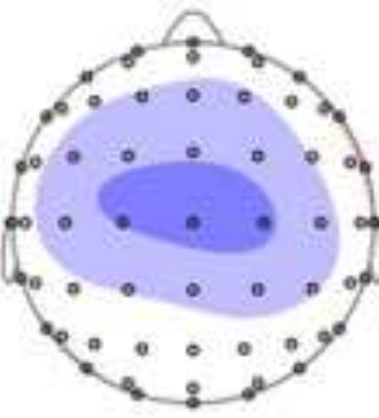

FRN

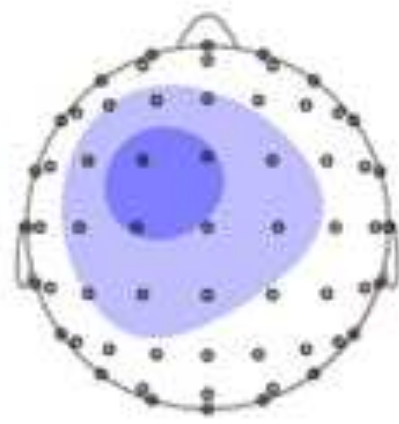

ERN

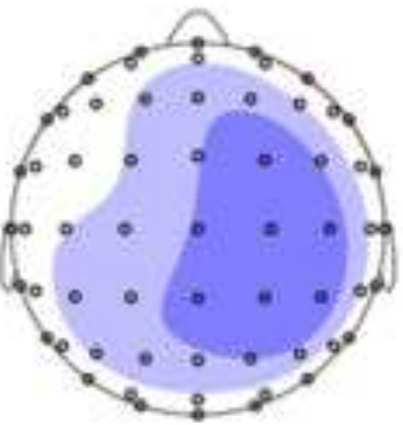

FRN

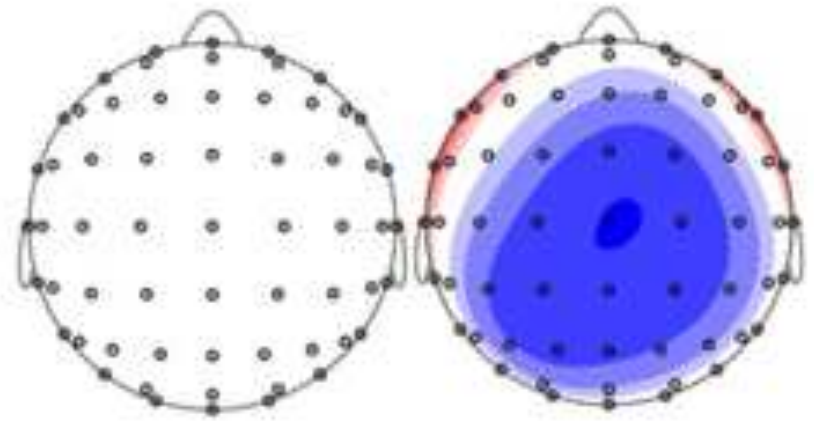

ERN

\section{Random}

